\title{
Portal bifurcation reconstruction using own hepatic vein grafts due to portal vein anomaly of the living donor for the patient with portal vein thrombosis
}

\author{
Akira Umemura, Hiroyuki Nitta, Takeshi Takahara, Yasushi Hasegawa, Hirokatsu Katagiri, \\ Shoji Kanno, Megumi Kobayashi, Taro Ando, Taku Kimura, and Akira Sasaki
}

\author{
Department of Surgery, Iwate Medical University Hospital, Yahaba, Japan
}

\begin{abstract}
A 57-year-old Japanese female was considered for living donor liver transplantation (LDLT) due to end-stage liver cirrhosis caused by primary biliary cholangitis with portal vein thrombosis (PVT) formation. A 26-year-old daughter of the patient was selected as a living donor; however, a computed tomography examination revealed trifurcated-type portal vein anomaly (PVA). Preoperative liver volumetry showed that the right lobe graft was necessary for the recipient; therefore, reconstruction of the portal vein bifurcation during LDLT was necessary. We planned to extract the recipient's own hepatic vein grafts after total hepatectomy, and these would be attached with anterior and posterior portal branches as jump grafts. We performed laparoscopic donor hepatectomy as usual, and the recipient's hepatic vein grafts were anastomosed on the bench. Then, the liver graft was inserted, and the hepatic vein reconstruction was routinely performed. We confirmed the alignment between the recipient's portal vein and the bridged hepatic vein graft of the liver graft's posterior branch, and anastomosed these two vessels. Moreover, we confirmed the front flow and expansion of the reconstructed posterior branch by declamping only the suprapancreatic side of the portal vein. The decision regarding the punch-out location was crucial. We confirmed the alignment between the reconstructed posterior branch and the bridged hepatic vein graft of the anterior branch, and anastomosed these two vessels employing the punched-out technique. In LDLT, liver transplant surgeons occasionally encounter living donors with PVA or recipients with PVT. Our contrivance may be useful when the liver graft needs reconstruction of portal vein bifurcation. (Ann Hepatobiliary Pancreat Surg 2020;24:533-538)
\end{abstract}

Key Words: Living donor liver transplantation; Portal vein thrombosis; Portal vein anomaly; Portal vein bifurcation; Interposition graft

\section{INTRODUCTION}

Portal vein thrombosis (PVT) is a critical preoperative problem in patients with end-stage liver disease, and the incidence of PVT is higher in patients with liver cirrhosis. ${ }^{1}$ Previous reports described that PVT is associated with an increased major complication rate and results in higher mortality. ${ }^{2}$ However, the presence of PVT is no longer considered an absolute contraindication for living donor liver transplantation (LDLT) because of the progress in surgical advancement and perioperative management after LDLT.

Portal vein anomaly (PVA) is also an important factor for a living donor candidate. ${ }^{3}$ Typically, in clinical practice, candidates with PVA tend to be excluded from the living donor due to difficulties in reconstruction and the presumed long ischemic time. In addition, reconstruction of portal vein bifurcation is extremely difficult, because it is necessary to simulate an appropriate alignment before reperfusion.

In this article, we report an extremely rare case of portal vein bifurcation reconstruction using the hepatic vein grafts of a recipient with PVT due to PVA in the living donor.

Received: May 4, 2020; Revised: June 22, 2020; Accepted: July 9, 2020

Corresponding author: Akira Umemura

Department of Surgery, Iwate Medical University Hospital, 2-1-1 Idaidori, Yahaba 028-3695, Japan Tel: +81-199077344, Fax: +81-196137111, E-mail: aumemura@iwate-med.ac.jp

Copyright (C) 2020 by The Korean Association of Hepato-Biliary-Pancreatic Surgery

This is an Open Access article distributed under the terms of the Creative Commons Attribution Non-Commercial License (http://creativecommons.org/ censes/by-nc/4.0) which permits unrestricted non-commercial use, distribution, and reproduction in any medium, provided the original work is properly cited. Annals of Hepato-Biliary-Pancreatic Surgery - pISSN: 2508-5778 - elSSN: 2508-5859 


\section{CASE}

A 57-year-old Japanese female was referred to Iwate Medical University Hospital to be considered for LDLT due to end-stage liver cirrhosis caused by primary biliary cholangitis. She had several episodes of ascites accumulation and a computed tomography (CT) scan revealed PVT (Fig. 1). Routine laboratory investigations showed serum total bilirubin, aspartate aminotransferase, alanine aminotransferase, albumin, creatinine, and sodium levels of $2.8 \mathrm{mg} / \mathrm{dl}, 70 \mathrm{IU} / \mathrm{L}, 26 \mathrm{IU} / \mathrm{L}, 1.7 \mathrm{~g} / \mathrm{dl}, 0.6 \mathrm{mg} / \mathrm{dl}$, and $140 \mathrm{mmol} / \mathrm{L}$, respectively, a prothrombin time international normalized ratio of 1.80 , and a platelet count of $8.4 \times 10^{4} / \mu 1$. Therefore, her Child-Pugh and end-stage liver disease scores were 10 and 12, respectively. From these work-up examinations, we concluded that her prognosis would worsen if she does not undergo liver transplantation as soon as possible. Furthermore, a PVT had already formed due to severe portal hypertension. Thus, we attempted to choose a living donor candidate among her relatives.

A 26-year-old daughter of the patient volunteered to be the living donor. Routine work-up examinations revealed that the daughter was immunologically ideal; however, CT examination showed a replaced right hepatic artery and trifurcated-type PVA (Fig. 2). In addition, drip infusion cholecystocholangiography $\mathrm{CT}$ also revealed the trifurcated-type main branches of the hepatic bile duct and the posterior hepatic bile duct overhung the supraportal side of the posterior portal vein branch. The preoperative liver needle biopsy revealed no noticeable histopathological change. Using liver volumetry, the right lobe volume of the donor was estimated to be $70.0 \%$ of her own whole liver volume, and the graft volume/recipient body weight ratio and percentage of graft volume/standard liver volume were 1.17 and $60.0 \%$, respectively (Table 1).
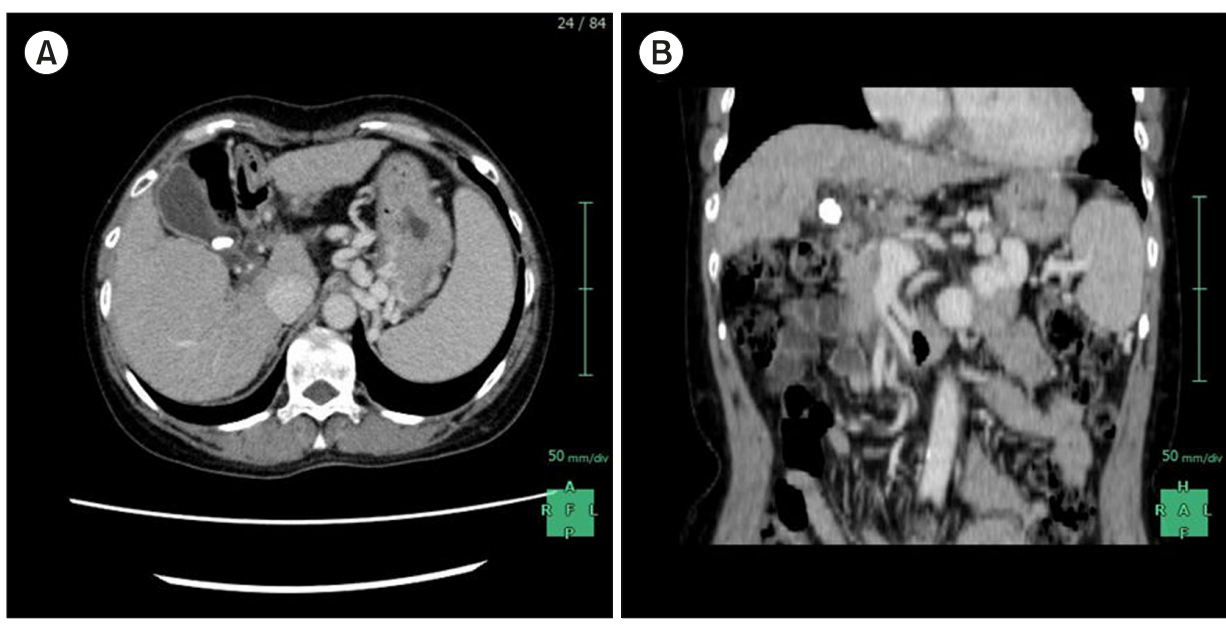

Fig. 1. (A) An Enhanced CT examination revealed that the PVT was formed at the hepatic hilum. (B) In coronal view, the PST started to form after confluence of the splenic vein.
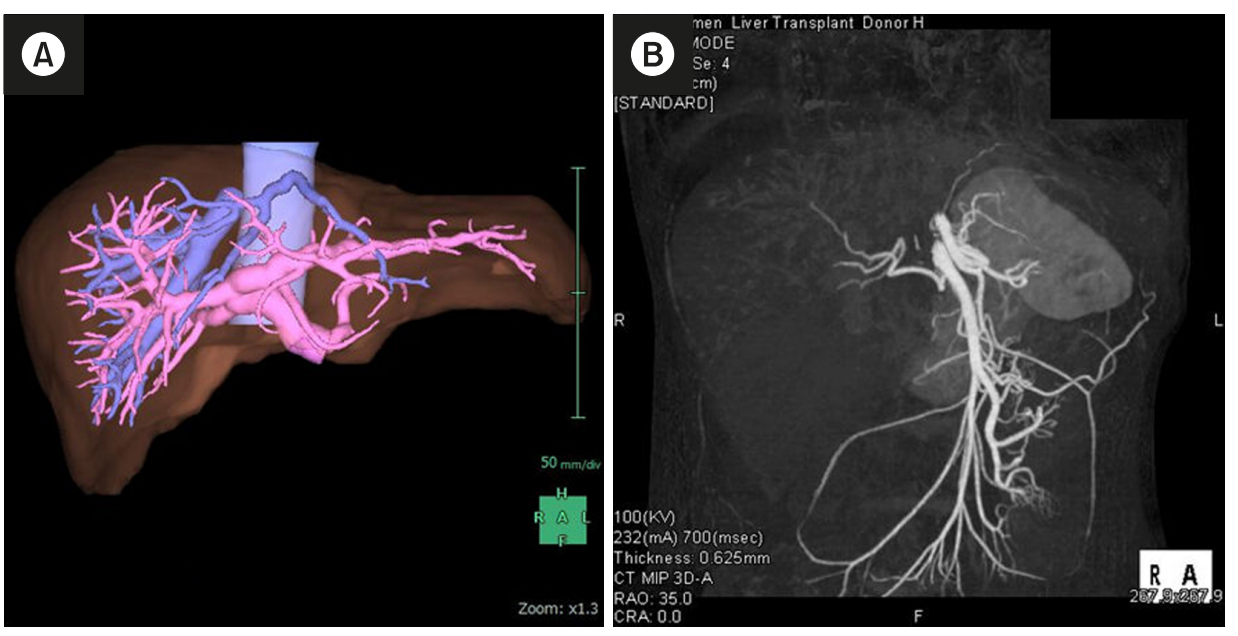

Fig. 2. (A) Preoperative simulation of the living donor revealed her type-III PVA. (B) A three-dimensional CT examination also revealed a replaced right hepatic artery. 
Other appropriate candidates of the living donor could not be found among her relatives; therefore, we planned to perform LDLT. However, portal vein reconstruction posed a critical problem, because the recipient had PVT, and the donor's right lobe graft would have two portal vein lumens due to PVA. This preoperative simulation helped us realize that the recipient's hepatic vein grafts should be extracted as long as possible after total hepatectomy and that these hepatic veins should be attached with the anterior and posterior portal branches of the liver graft. We also prepared to extract a great saphenous vein in case the long hepatic vein grafts could not be extracted. We applied laparoscopic donor right hepatectomy and transected the right hepatic duct after intraoperative cholangiography employing indocyanine green fluorescence imaging (Fig. 3A). The portal branches were detected just

Table 1. Preoperative estimations of the recipient's and donor's characteristics

\begin{tabular}{lcc}
\hline \multicolumn{1}{c}{ Variable } & Recipient & Donor \\
\hline Physical findings & & \\
Height $(\mathrm{cm})$ & 151.0 & 153.0 \\
Body weight $(\mathrm{kg})$ & 51.5 & 40.5 \\
Body surface area $\left(\mathrm{m}^{2}\right)$ & 1.46 & 1.32 \\
Standard liver volume $(\mathrm{ml})$ & 1007 & \\
Blood type (ABO, Rh) & $\mathrm{O}+$ & $\mathrm{O}+$ \\
CT volumetry & & \\
Whole liver volume (ml) & & 603 \\
Right lobe (ml) & & 70.0 \\
Graft volume/Whole liver volume (\%) & & 1.17 \\
Graft volume/Recipient body weight & & 60.0 \\
Graft volume/Standard liver volume (\%) & & \\
\hline
\end{tabular}
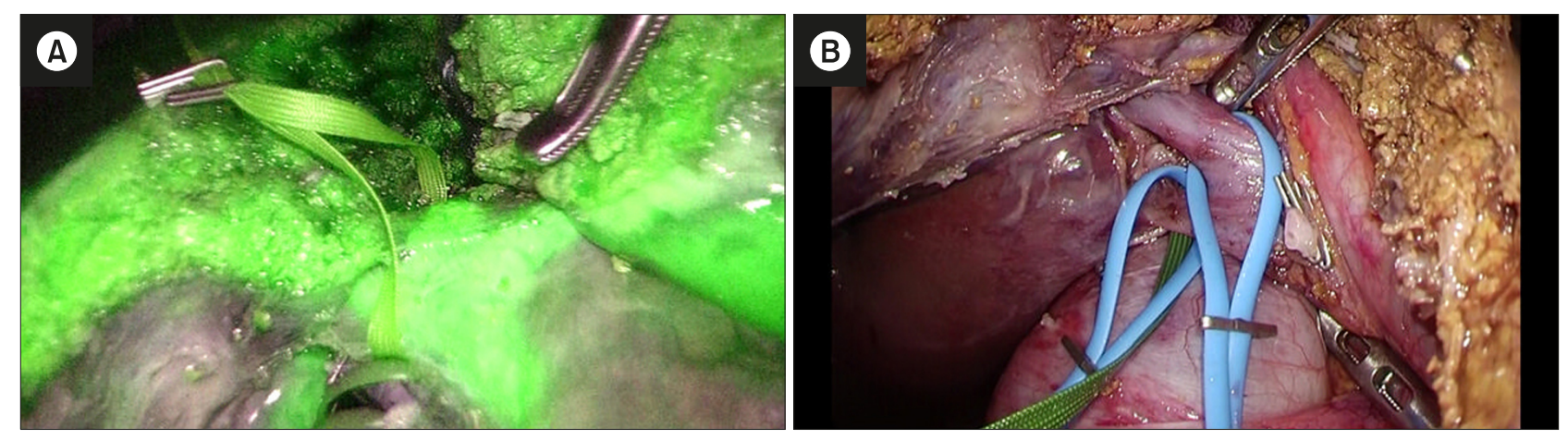

Fig. 3. We performed laparoscopic donor right hepatectomy. (A) We performed intraoperative cholangiography using indocyanine green fluorescence imaging before transection of the right hepatic duct. (B) Anterior and posterior portal branches were individually taped as preoperative simulation. as predicted in the preoperative simulation; therefore, we transected both branches of the portal vein after test clamping and intraoperative sonography (Fig. 3B). On the other hand, the recipient's total hepatectomy was routinely transected without PVT at the suprapancreatic side. Two long hepatic vein grafts were extracted from her own liver specimen on the bench, and then, these extracted hepatic vein grafts were anastomosed to the portal branches after usual graft perfusion. The right lobe graft was inserted, and reconstruction of the hepatic vein was routinely performed. We confirmed the alignment between the recipient's portal vein and the bridged hepatic vein graft of the graft's posterior branch, and anastomosed these two vessels (Fig. 4A). Then, we confirmed the alignment between the reconstructed posterior branch and the bridged hepatic vein graft of the graft's anterior branch, and anastomosed these two vessels employing the punched-out technique (Fig. 4B). We started blood reflow of the transplanted liver graft, and intraoperative sonography revealed good hepatopetal flow of the reconstructed portal vein branches (Fig. 4C). Schemas of these procedures are shown in Fig. 5. Finally, hepatic artery and biliary tract reconstructions were performed. Her postoperative recovery was almost satisfactory without major complications, and postoperative enhanced CT examination revealed the patency of the reconstructed portal branches (Fig. 6). She was discharged from our hospital on the 67th postoperative day after physical rehabilitation for daily life after discharge. performed, and the main portal vein was successfully 

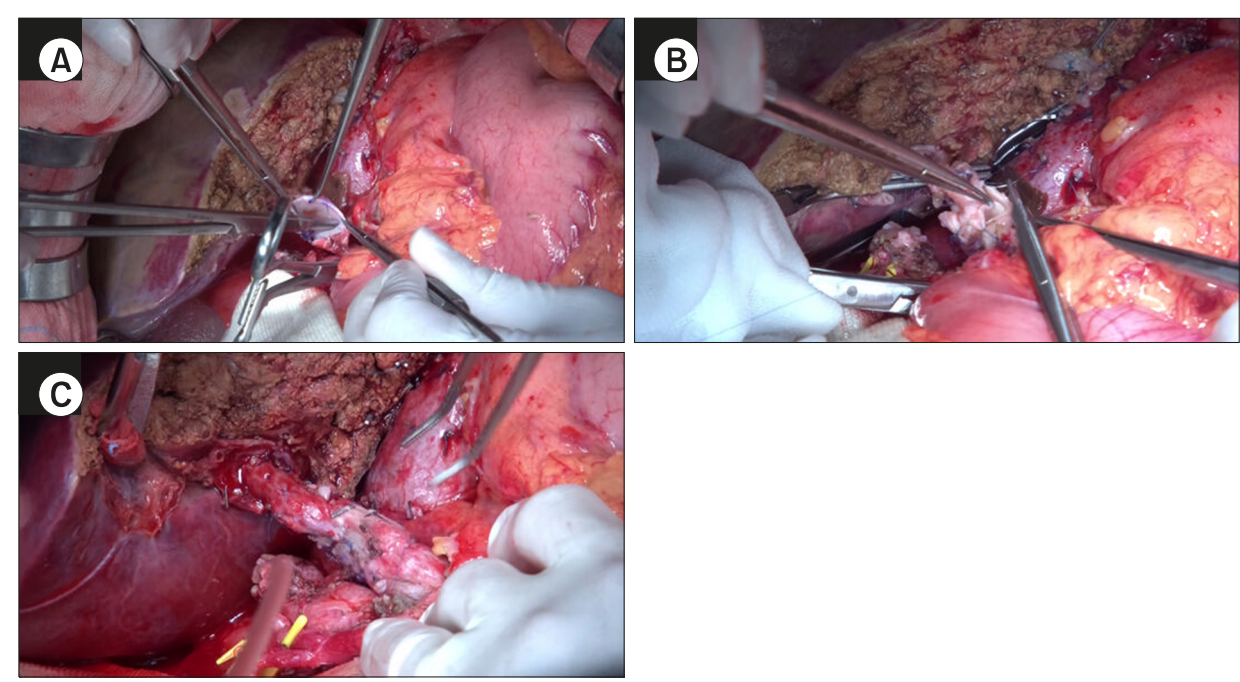

A

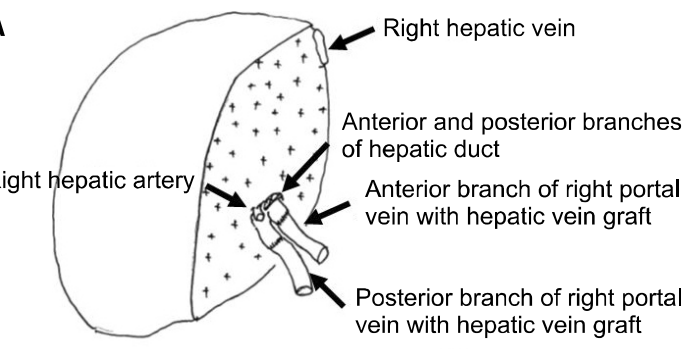

B

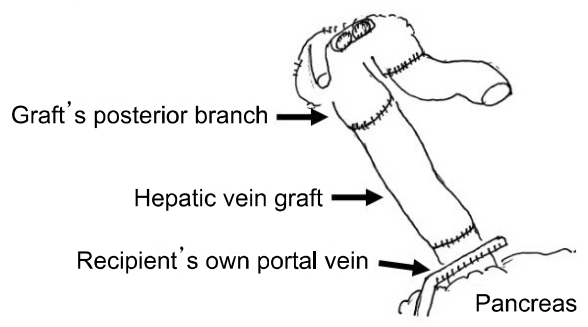

C

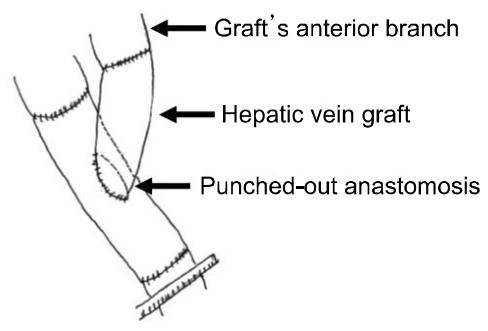

Fig. 5. Schemas of portal vein bifurcation reconstruction is shown. (A) Two long hepatic vein grafts were anastomosed to the portal branches on the bench. (B) The posterior branch with hepatic vein graft was anastomosed to the recipient's main portal vein. (C) Punched-out anastomosis of the anterior branch was performed on the interposed hepatic vein graft.

\section{DISCUSSION}

Recipient work-up examinations typically reveal PVT, which poses one of the most intricate problems in LDLT. Some case series have already confirmed that patients
Fig. 4. Surgical procedure of portal vein bifurcation reconstruction is shown. (A) The posterior branch with interposition hepatic vein graft was anastomosed with recipient's portal vein. (B) We confirmed the alignment between the reconstructed posterior branch and the bridged hepatic vein graft of the graft's anterior branch, and anastomosed these two vessels employing the punched-out technique. (C) The reconstructed portal vein bifurcation supplied a good hepatopetal flow.

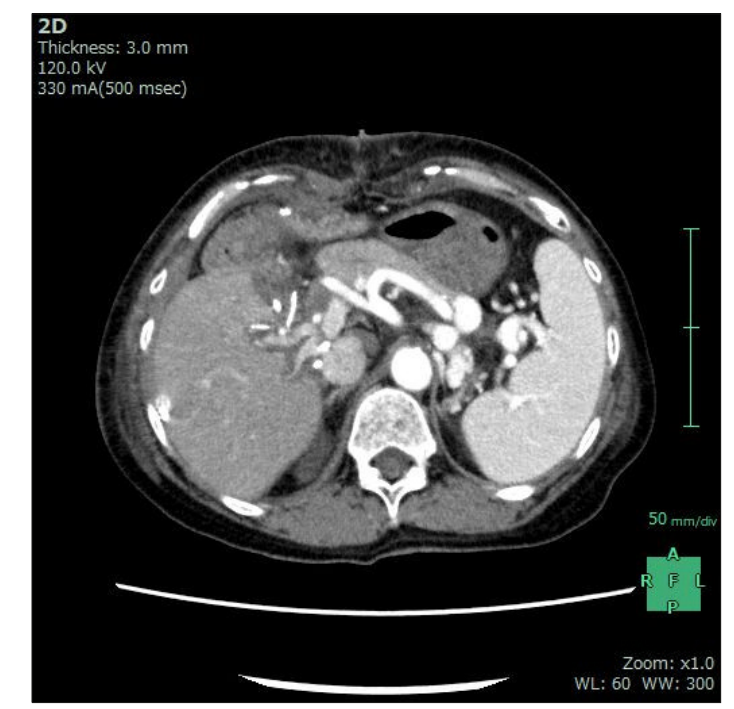

Fig. 6. A postoperative protocol CT revealed patency of reconstructed portal vein bifurcation without any complications.

with PVT required more complex surgeries in LDLT compared to deceased donor liver transplantation. However, Hibi et al. ${ }^{4}$ reported that comparable long-term outcomes were achieved in patients with complete PVT as long as the physiological portal inflow was restored. ${ }^{5}$ In LDLT, securement of physiological portal inflow might be the priority for the recipient, because non-physiological portal inflow is correlated with a higher complication rate brought about with issues such as re-PVT and gastrointestinal bleeding. ${ }^{5}$ In this case, preoperative simulation revealed that we would be able to transect the recipient's portal vein at the suprapancreatic side; therefore, it was not necessary to employ a mesoportal jump graft and per- 
form non-physiological portal inflow.

On the other hand, we were confronted with the living donor's PVA. Previous reports revealed that PVA is observed in $20-35 \%$ of the population. ${ }^{3,5}$ Cheng et al. ${ }^{5}$ classified intrahepatic portal vein variations into five different types, and our living donor had type-III PVA, in which the right posterior portal vein arises directly from the main portal vein as its first branch. Type-III PVA is the second commonest variant, observed in $0.7-3.0 \%$ of the population. $^{3}$ It is very difficult to perform intraoperative clamping, especially during laparoscopic donor hepatectomy. We invented laparoscopic donor hepatectomy employing the Glissonean approach; ${ }^{6}$ however, using this approach for PVA is impossible due to the different branchings of the vessels and hepatic ducts. We employed individual taping and transection for this critical laparoscopic donor hepatectomy. Type-III PVA also affects the recipient's portal vein reconstruction, because it is expected that two portal vein anastomoses are to be performed on two different veins in the recipient. If the recipient did not have PVT, we might consider extracting and using her own portal vein bifurcation graft, because portal vein reconstruction by single anastomosis after the insertion could shorten the warm ischemic time. ${ }^{7}$ However, we could not use her own portal vein bifurcation graft due to PVT. In this case, Reversed middle and left hepatic vein graft was not also suitable because the alignment and the angle of the bifurcation was limited; therefore, we planned to extract her own hepatic vein grafts and attach these hepatic veins with the anterior and posterior portal branches of the liver graft. Certain factors, such as discrepancy of the vessel diameters, the top and bottom parts of the vessels, tension after anastomosis, and alignment of the anastomosed portal vein, are crucial for portal vein reconstruction. We cautiously checked the alignment between the main portal vein of the recipient and the hepatic vein jump graft of the posterior branch, because the posterior branch should be straight for the end-to-side anastomosis of the anterior branch. Moreover, we confirmed the front flow and expansion of the reconstructed posterior branch by declamping only the suprapancreatic side of the portal vein. It was very crucial to decide the location of the punch out. We surmised that the tension and angle of the end-to-side anastomosis of the anterior branch should not be very tight, because liver regeneration after
LDLT would change the alignment of the anterior branch. Therefore, a slight slackening of the reconstructed anterior branch appeared to be the best option to avoid kinking.

In this paper, we described an extremely rare case of portal bifurcation reconstruction using the recipient's own hepatic vein grafts due to PVA in the living donor and the fact that the recipient suffered from PVT. Careful preoperative simulation and alignment checking of the posterior branch after insertion are mandatory to perform this procedure safely and quickly. Stagnation of the portal vein reconstruction after the insertion will prolong the warm ischemic time and influence graft survival negatively. In LDLT, as liver transplant surgeons occasionally encounter living donors with PVA, our contrivance may be useful when the liver graft needs reconstruction of the portal vein bifurcation.

\section{CONFLICT OF INTEREST}

The authors have no conflict of interest to disclose.

\section{ORCID}

Akira Umemura: https://orcid.org/0000-0003-4181-7159 Hiroyuki Nitta: https://orcid.org/0000-0003-1606-4773

Takeshi Takahara: https://orcid.org/0000-0003-4800-955X

Yasushi Hasegawa:https://orcid.org/0000-0002-4990-0419

Hirokatsu Katagiri: https://orcid.org/0000-0002-4567-6106

Shoji Kanno: https://orcid.org/0000-0002-7678-3078

Megumi Kobayashi: https://orcid.org/0000-0002-7688-4805

Taro Ando: https://orcid.org/0000-0003-2792-6930

Taku Kimura: https://orcid.org/0000-0002-0059-6372

Akira Sasaki: https://orcid.org/0000-0002-1346-5312

\section{AUTHOR CONTRIBUTIONS}

Conceptualization: AU, HN, TT, YH. Data curation: HK, SK, MK, TA, TK. Formal analysis: AU, HN, TT, YH, HK, SK, MK, TA, TK, AS. Funding acquisition: AU. Methodology: HN, TT, YH. Project administration: AU, HN, TT, YH, HK, SK, TA, TK. Visualization: AU. Writing - original draft: AU. Writing - review \& editing: HN, TT, YH, AS. 


\section{REFERENCES}

1. Song S, Kwon CH, Kim JM, Joh JW, Lee SK. Single-center experience of living donor liver transplantation in patients with portal vein thrombosis. Clin Transplant 2016;30:1146-1151.

2. Lendoire J, Raffin G, Cejas N, Duek F, Barros Schelotto P, Trigo $\mathrm{P}$, et al. Liver transplantation in adult patients with portal vein thrombosis: risk factors, management and outcome. HPB (Oxford) 2007;9:352-356.

3. Iqbal S, Iqbal R, Iqbal F. Surgical implications of portal vein variations and liver segmentations: a recent update. J Clin Diagn Res 2017;11:AE01-AE05.

4. Hibi T, Nishida S, Levi DM, Selvaggi G, Tekin A, Fan J, et al. When and why portal vein thrombosis matters in liver transplantation: a critical audit of 174 cases. Ann Surg 2014;259:760-766.

5. Cheng YF, Huang TL, Lee TY, Chen TY, Chen CL. Variation of the intrahepatic portal vein; angiographic demonstration and application in living-related hepatic transplantation. Transplant Proc 1996;28:1667-1668.

6. Hasegawa Y, Nitta H, Takahara T, Katagiri H, Kanno S, Sasaki A. Pure laparoscopic living donor hepatectomy using the Glissonean pedicle approach (with video). Surg Endosc 2019;33: 2704-2709.

7. Song JL, Wu H, Yang JY. Laparoscopic donor right hepatectomy in a donor with type III portal vein anomaly: a case report. Medicine (Baltimore) 2019;98:e16736. 